\title{
Job Stress, Sector of Work, and Shift-Work Pattern as Correlates of Worker Health and Safety: A Study of a Manufacturing Company in Ghana
}

\author{
Collins Badu Agyemang ${ }^{1}$, Joseph Gerald Nyanyofio ${ }^{1} \&$ Gerald Dapaah Gyamfi ${ }^{1}$ \\ ${ }^{1}$ Faculty of Management, Department of Business Administration, University of Professional Studies, Accra, \\ Ghana
}

Correspondence: Collins Badu Agyemang, Faculty of Management, Department of Business Administration, University of Professional Studies, Accra, Ghana. E-mail: agyemang.collins@yahoo.com

Received: March 13, 2014

Accepted: May 4, $2014 \quad$ Online Published: June 22, 2014

doi:10.5539/ijbm.v9n7p59

URL: http://dx.doi.org/10.5539/ijbm.v9n7p59

\begin{abstract}
Worker health and safety must be viewed as a basic organizational policy and even a right. In view of this, the study sought to ferret out the extent to which job stress, sector of work and shift work pattern correlates with worker health and safety in a manufacturing company in Ghana. Using cross-sectional survey design, data were collected from 120 employees using purposive and stratified sampling techniques. Three hypotheses were tested using Pearson Product Moment Correlation and Independent Sample ' $t$ ' test. A significant correlation was found between employee stress and worker health and safety. Thus an increase in employee stress will lead to a decrease in worker health and safety and vice versa. No significant difference was found in the level of health and safety among workers in the production sectors compared to workers in the non-production sectors. The study established that employees who go through shift work will experience lower health and safety compared to employees who do not go through shift-work. The present study is one of the foremost research attempts on sector of work and worker health and safety with a comparative approach using sample from the manufacturing setting in Ghana. The researchers recommend that employers take necessary steps towards introducing a culture where a stress prevention plan exists and worker health and safety is prioritised. The researchers reckon this as necessary step for policy implementation as worker health and safety policies are fragmented in most developing countries.
\end{abstract}

Keywords: Stress, sector of work, shift-work pattern, worker health and safety, Ghana

\section{Background to the Study}

Research on health and safety has been consistently viewed differently for decades both as a humanitarian and a Corporate Social Responsibility (CSR) by most employers (Adonteng, 2013). However, globally, most researchers tend to focus on the antecedents of worker health and safety predominantly among health care providers (Landsbergis, 2006; Letvak, 2005). Some researchers of stress (e.g. Turk, 2002; Willner, 1997) as antecedents of health and safety veer toward reviewing or examining the existing models in an attempt to propose another.

Stress is the result of any emotional, physical, social, economic or other factor that requires a response to change (Alberta, 2008). Job stress results from the interaction of the worker and the conditions of work (Durand, 2003). Views differ on the importance of worker characteristics versus working conditions as the primary cause of job stress. These differing viewpoints are important because they suggest different ways to prevent stress at work (Durand, 2003). According to Spurgeon, Harrington and Cooper (1997) existing data on stress relate largely to situations where working hours exceed 50 a week and there is a lack of information on hours below this level, and in most cases of direct relevance to the European community proposal. This therefore suggests that the extent of applicability of such findings to other regions such as Africa and Asia is limited. Other investigations of stress relate to shift work where a range of modifying factors is likely to influence the level and nature of health and performance outcomes (Spurgeon, Harrington \& Cooper, 1997). Stress is the second most reported work-related health problem and the number of people suffering from stress-related conditions caused or made worse by work is likely to increase (Fimian, Fastenau, \& Thomas, 2009). Although the importance of individual differences cannot be ignored, scientific evidence suggests that certain working conditions, such as excessive 
workload demands, section of operation and conflicting expectations, are stressful to most people (Luk, 2009). Such evidence argues for a greater emphasis on working conditions (for example, noise, lighting, smells, heat), shift work, long hours, travel, risk and danger, new technology, work overload and work underload as the key source of workplace stress, and for job redesign as a primary prevention strategy (Luk, 2009). In the researchers' opinion, most of these working conditions (for example, noise, lighting, smells, heat) are experienced most by employees at the production departments compared to those outside that terrain. According to Bellani, Furlani, Gechi and Pezzolta (1996) factors intrinsic to the job, role in the organization, personality and coping strategies, relationship with employees, career development, home and work interface.

A direct effect on stress outcomes could be psychological and/or physical health problems (Patton, 1990;Plante, 1995). The demands on the individual in the workplace reach out into the homes and social lives of employees. Long, uncertain or unsocial hours, working away from home, taking work home, high levels of responsibility, job insecurity and job relocation may all adversely affect family responsibilities and leisure activities (Young \& Ogden, 2008). Patton (1990) argues that the sources of stress differs from one department of the organization to the other and thus two people working in the same organization but in different department will experience different levels of stress and it is envisioned that there might be variations in the health and safety of the employees as wellStudies (e.g., Wu, Wang, \& Lan, 2007) show that stressful working conditions are actually associated with increased absenteeism, tardiness, and intentions by workers to quit their jobs-all of which has a negative effect on the bottom line. Studies indicate that policies benefiting worker health also benefit a successful organization (Young \& Ogden, 2008). Work stress has been shown to result in job dissatisfaction, burnout (physical, emotional, and mental exhaustion), staff turnover, occupational illness and injuries, reduced mental health, depression, and even suicide (Lundstrom, 2002). Workplace stress and mental ill health often go hand in hand, so it's important to look at the nature of the relationship between the two, in particular how workplace stress can act as a trigger to mental health problems (Bennett \& Kelaher, 2004). According to Costa (1997), nearly three in every ten employees will have a mental health problem in any one year, the great majority of which will be anxiety and depressive disorders resulting from workplace stress. Half of all days lost through mental ill health are due to anxiety and stress conditions. The severity of the statistics warrants a study in this area in especially in Sub-Saharan in an attempt to ensure regional literature balance. Durand (2003) established a strong relationship between job stress and a variety of ailments such as mood and sleep disturbances, upset stomach and headache, and disturbed relationships with family and friends.

According to Alberta (2008), some stress may be necessary to serve as some form of challenge or positive stress but may lead to some mental and physical changes when intensified and cannot be handled. Many studies have suggested that psychologically demanding jobs that allow employees little control over the work process increase the risk of cardiovascular disease (Bennett \& Kelaher, 2004). There is a growing concern that stressful working conditions interfere with safe work practices and set the stage for injuries at work (Bennett \& Kelaher, 2004). The changing world of work is making increased demands on workers, through downsizing and outsourcing, the greater need for flexibility in terms of function and skills, increasing use of temporary contracts, increased job insecurity and work intensification (with higher workload and more pressure), and poor work-life balance.

Job stress has been a relatively neglected area of research among industrial/organizational psychologists (Beehr \& Newman, 2006) and this situation is especially grave in Sub-Saharan Africa and for that matter Ghana. The rate of industrialization in Ghana, as a strongly emerging middle level income status economy and a spot light within the Sub-Saharan terrain is impressing and this ascendancy and has led to larger percentage of the Ghanaian workforce being exposed to workplace physical, chemical, biological and psychological stressors. Not to mention the fierce competition among organizations within industries that persistently calls for demonstrating citizenship behaviour (Badu \& Asumeng, 2013). It is however fascinating to ask whether a nation like Ghana has got a system for anticipating and monitoring the likely effects of these strides on employees? Empirical research interest on health and safety has received very little attention from the Ghanaian outlook.

It is intriguing to know that not much literature is there from the Ghanaian perspective on this humanitarian area and if there is, most are unempirical reports of tasked agencies mandated to regulate such occurrences on policy lines. Most production organizations are no exception when it comes to organizational stress. Given the wide range of potential and/or actual undesired events associated with the myriad of work groups in Ghana, there is the need to have a comprehensive provision for occupational safety and health standards and practice in the nation, with an unflinching national leadership, support and commitment. This can arguably be done properly on the basis of research grounding. Undertaking an empirical study to unravel the predictors of health and safety from the researchers' scrutiny should spur and stimulate empirical research interest. This attempt is aimed at igniting the 
attention of policy makers about the need to constitute a national body, policy or process that governs Occupational Safety and Health Management in Ghana as this is absent presently. In an attempt to bridge the above gaps in global and regional literature, the present study focuses on job stress and sector of operation as correlates of employee health and safety in a manufacturing company of Ghana.

\subsection{Objectives of the Study}

Specifically, the following objectives were achieved:

1) To ferret out the relationship between organizational stress and worker health and safety.

2) Ascertain whether there will be any significant difference in employee health and safety due to their department/sector of work.

3) To find out whether employees who go through shift work will experience lower level of health and safety compared to those who do not go through shift work.

\section{Underpinning Theories}

Martens Multidimensional Health theory (Martens, 2001) and Environmental Stress Model (Baum, Singer \& Baum, 1981) formed the basis for the present study. Multidimensional Health Theory (Martens, 2001) is based upon the notion that psychological health is multidimensional in nature, composed of a cognitive component and a somatic component. Multidimensional theory specifically hypothesizes two things (1) a negative linear relationship exists between cognitive state in terms of stress, depression, anxiety and health, and (2) An inverted-U relationship exists between somatic stress, depression, anxiety and health. According to the multidimensional theory, in the case of cognitive state anxiety, stress and depression, as anxiety increase, performance and health begins to deteriorate. In the case of somatic state anxiety, as anxiety, stress, and depression increase athletic performance and health increases to a certain level, and after that level athletic performance begins to drop. Somatic state anxiety forms an inverted U-shape. On a similar note the environmental stress model posits that elements within the environment (in this case the work environment) cause stress. Stress is an intervening variable, which is defined as a reaction to these elements. The reaction involves emotional, behavioural and physiological. According to Baum, Singer and Baum (1981) who are the major proponents of this model, these components do not occur in isolation. The behavioural and emotional constitute the psychological aspect. The psychological and physiological then becomes a unitary factor within the environmental stress model. Based on the assumptions of the above theories, the present study examined job stress, department of work as predictors of health and safety of employees

\subsection{Related Studies}

Piko (1999) stated that the issue of occupational stress among employee is a major concern in health policy and he found the level of stress experienced by employee to be dependent on the sector of the organization which they worked. Chan, Lai, Ko, and Boey (2000) found a significantly negative relationship between stress and the amount of accident that occurred among six professional groups including physicians, engineers, life insurance agents, lawyers, nurses, and teachers. Zamanian, Kakooei, Ayattollahi, Choobineh, and Seraji, (2007) conducted a study to determine the prevalence of mental disorders among shift workers. $45.4 \%$ of employees were found to suffer from mental disorders and this was more common among females. Similarly, anxiety and somatic symptoms were more prevalent than other types of mental disorders ( 43.2 and $34.5 \%$, respectively). The prevalence of depression and social dysfunction were 11.2 and $79.5 \%$, respectively. Shift work was significantly associated with lower health than non-shift workers. A study by Adjei (2009) on work-stress and its management among senior staff of the University of Cape-Coast in Ghana, discovered that respondents experienced both physiological and psychological symptoms of stress. It was also found that workload, lack of recognition for good effort, and inadequate opportunities for career development were prominent stressors among the respondents.

Iqbal and Kokash (2011) conducted a study to assess the level of stress among employees working in the production sectors and administration sectors. The study was conducted to explore the production and administrators perception towards occupational stress using established questionnaire, data collected from five departments in the organization. Research findings indicated a significantly higher level of stress among employees in the production sector compared to the administrators. Sanders (2009) assessed the perceptions of stress among employees in the production sectors and administrators. The study indicated no significant difference in the level of employee health among administrators and production members even though production members experienced higher level of stress compared to administrators. 
Gmelch, Wilke and Lovrich (1996) investigated identifiable patterns of stress and health issues among the different sectors of a manufacturing company. From a sample of 12 organizations, 920 employees took part in the study. The response rate was $75.28 \%$. The multidimensionality of the 45 -item Faculty Stress Index, investigated through factor analysis, resulted in five distinct dimensions of perceived stress: reward and recognition (55\% common variance); time constraints $(12 \%$ common variance); departmental influence ( $7 \%$ common variance); professional identity (6\% common variance); and customer interaction $(6 \%$ common variance). Each factor was also analyzed according to professional and personal characteristics, and the analysis resulted indicated a significantly higher level of stress among employees in production sectors for almost all the five distinct dimensions of the perceived stress. Those in the production sectors were also found to experience lower levels of psychological health and safety.

Reeves, Newling-Ward and Gissane (2004) revealed that, night workers did not eat more than day workers, but ate smaller meals and snacks over a greater time frame. Night shift worker revealed significant difference $(p<0.05)$ in food intake patterns on work and rest days, while day workers did not. Because shift work is a factor in the timing of food consumption and typically there is a lack of good food facilities at night, it suggested that this has the potential to cause difficulties in the establishment of healthy eating patterns. This study also failed to have a control group hence the need for a comparison between a day shift and a night shift. Akerstedt (2007) examine the time of day that one work and its effect on sleep and performance and found that night work induces more errors in performance and decrease the ability to concentrate. Demi (2006) did a study among nurses working in different departments in a hospital and analyses showed that nurses experiencing stress from their workplace were significantly more likely to use wishful thinking, painful problem solving, and avoidance as coping strategies, whereas stress originating from patient care was more likely to be dealt with using positive appraisal and acceptance. Nurses working overloaded sectors were found to experience higher levels of stress than those whose workload was not higher. To the extent of the researchers search and as reviewed above, none of them were conducted in the sub-Saharan regions (Ghana inclusive indicating that health and safety literatures are very scarce in Ghana and thus the need to undertake the study in Ghana. Moreover, the results of the studies have been inconsistent. Some of the studies reviewed (e.g., Gmelch, Wilke \& Lovrich, 1996; Sander, 2009) have also indicated no significant relationship between stress and health; others (e.g., Iqbal \& Kokash, 2011) have found a significant positive relationship between stress and health. Based on the above theories and literature reviewed, the present study examines job stress, department of work and shift pattern as predictors of worker health and safety in Ghana.

Giovani (1996) found that shift work impacted negatively on employees' health and well-being. However, Giovani (1996) suggested some other variables such as work situations and social conditions at work place can affect employee health and safety. One of such variables may include the section of operation and its impact on health and wellbeing of employees. Landsbergis (2003) argued that there seem to be little research evidence on the recent trends in the organization of work and the effect on worker health. It has been about a decade since Landsbergis (2003) threw that challenge to scholars but the available evidence has not change much in the Sub-Saharan region with Ghana inclusive.

\subsection{Statement of Hypotheses}

Based on the objectives and literature reviewed above, the study tested the following hypotheses:

1) There will be a significant negative relationship between employee stress and health and safety.

2) Workers in the production sectors will experience lower health and safety compared to workers in the non-production sectors from the Ghanaian setting.

3) Employees who go through shift work will experience lower health and safety compared to employees who do not go through shift work.

\subsection{Operational Definitions}

Stress: experiencing strong physical pressures or difficulties as indicated on the score on the stress scale.

Higher stress: a score of $56-85$ as measured on the stress scale

Low stress: a score of $17-55$ as measured on the stress scale

Health: The condition of an individual's body and the extent to which it is prone to or free from illness.

Safety: The extent to which a worker is free from potential harm or injury.

Sector: Sector is used interchangeably with department or section to mean an employee's area of operation. 
Production section: working in core production point (beverage and cereal sectors) of the organization.

Non-production section: employees in logistics, distribution centre and central administration units.

\section{Method}

As suggested by Wiersma and Jurs (2005), the 'methods and procedures is really the heart of the research, activities should be described with as much detail as possible and the continuity between them should be apparent' (p. 416).

\subsection{Research Setting}

The manufacturing industry used for the study produces beverages, cereals and milk in large sums to meet the demands of its consumers. Such an organization with high standing reputation is likely to be concerned with issues of Stress and Health and Safety of employees. There are however, two groups of employees namely; production and non-production employees. Those in the production are expected to meet specific targets set for each production department every week in relation to the amount or quantity of beverages, cereals and milk to be produced. Most employees are left with the option to work overtime to meet these goals. Supervisors may also be under pressure to produce products in their batches with no shortfalls in quality. Such items are either reworked on or destroyed depending on the state, this to a greater extent puts pressure on not only supervisors but also the other production team members since they are to restart the whole process. The management team and other senior staff members (most of them in supervisory or administrative positions) are required to work straight day from 8:30am to 16:30pm (Greenwich Meridian Time). Other employees (most of whom are junior staff) run shift-work for morning, afternoon and evening, that in the period of 5:00am to 2:00pm, 2:00pm to 8:00pm and 8:00pm to 5:00am respectively. The researchers considered this manufacturing organization to be most suitable setting for the study.

\subsection{Research Design}

The study design was a cross sectional survey. Respondents were assessed by taking a cross-section of the occurrences at a given time and analysing the cross-section carefully. This method facilitates asking a large number of workers their opinions in a relatively less time and cost effective manner (Oppenheim, 1992).

\subsection{Population}

Since its establishment, the manufacturing company has significantly stimulated the Ghanaian economy with a dynamic, well-trained and dedicated workforce, manufacturing and marketing well-known brands and so ensuring the commitment level of its employees have a direct influence on the Ghanaian economy. The current population size of the manufacturing company is about 730. This population was chosen for the study because the researchers found it appropriate since the industry is noted to be a renowned organization that is involved in mass production of beverages, cereals and milks alongside other non-productive activities, with different segments and have garnered initiative for worker wellbeing. For this reason the health and safety conditions of workers was of great concern to the researchers.

\subsection{Sample}

According to Buckingham and Saunders (2004) a sample may consist of a tiny fraction of the whole target group, but provided it is selected carefully and methodologically, it can provide remarkably accurate estimates of the parameters of the whole population. Out of the general population, 120 participants were sampled from the various departments stated (cereals, beverages, logistics and central distribution centre) of both the production and non-production sections for the study, 30 participants were selected from each of these departments (beverages, cereals, logistics department, and central distribution centre). The sample comprised management, senior and junior staff members.In line with Tabacknick and Fidell's (2007) recommendation, the minimum sample size for parametric testing should be $104+\mathrm{M}$ for testing individual predictors (where $\mathrm{M}$ is the number of predictors). Drawing from the above, the minimum sample size required to test for the individual variables should be 107 and above to be able valid conclusions. Table 1 below is a summary of the demographic characteristics of respondents. 
Table 1. Demographic characteristics of the respondents

\begin{tabular}{lll}
\hline Variable & Frequency & Percentage \\
\hline Sex & & \\
- Male $\quad 66$ & 55.0 \\
- Female & 54 & 45.0 \\
Sector & & \\
- Production & 66 & 55.0 \\
- Non-production & 54 & 45.0 \\
Rank & & \\
- Senior & 30 & 25.0 \\
- Junior & 90 & 75.0 \\
\hline
\end{tabular}

The demographic analysis revealed a male dominance (55\%) of the participants as against females (45\%). Moreover, $25 \%$ and $75 \%$ occupied senior managerial and junior managerial positions respectively. Most of these junior staff works in the production sectors where safety procedures are requisite.

\subsection{Sampling Technique}

Stratified random sampling technique combined with purposive sampling technique was used to obtain the sample. That is the, departments for the study was grouped into production and non-production sections with Beverages and Cereals making up the production section and Logistics, Central Distribution Centre and administration units making up the non-production section.

\subsection{Instruments}

Standardized questionnaires were used for collecting data for the study. The scales used were divided into three sections labelled 'A' to C'. The section ' $A$ ' measured the demographic data of workers such as sex, age, sector of working and number of years in service.

Section 'B' was an adapted version of the Job Stress scale.The scale consists of 10 test items measured on a five point Likert scale ranging from; (1)-often to (5)-never. The following items 3, 5, 6, 9, 13 and 17 were reversed scored. Some items on the scale includes, 'do you have to work very fast' and 'does your work demand too much effort'.

Gravesham Borough Council questionnaire was adapted to measure employee level of health and safety. The scale has 39 items measured on a three point scale that is yes, no or not applicable. The questionnaire has a cronbach alpha of .88. Some items on the scale includes: 'I normally feel sick', 'I seldomly cause accident'.

\subsection{Pilot Study}

To ascertain the reliability and the appropriateness of the two instruments, 30 participants consisting of 15 production workers and 15 non-production workers of a different but analogous manufacturing company were used and a split half procedure produced a reliability coefficient of 0.82 and 0.79 for job stress and health and safety questionnaires respectively. The possible minimum and maximum scores for the health and safety scale were 39 and 117 respectively with a higher score indicating a lower level of health and safety and vice versa.

\section{Results}

Data was analysed in two sections, the first section consisted of the demographic characteristics describing the frequency and percentage of all the variables employed in the study. The second section dealt with the inferential statistics for testing the various hypotheses.

\subsection{Hypotheses Testing}

Hypothesis 1: there will be a significant negative relationship between employee stress and health and safety. This hypothesis was analysed using the Pearson Product Moment Correlation because the relationship between two simple variables (stress, health and safety) were established. 
Table 2. Employee stress and worker health and safety

\begin{tabular}{lll}
\hline Measures & 1 & 2 \\
\hline Employee stress & - & $.319^{* *}$ \\
Health and Safety & - & - \\
\hline
\end{tabular}

Note. ${ }^{* *}$ Correlation is significant at the 0.01 level (1-tailed).

As shown in Table 2, the relationship between employee stress and health and safety was significant $(\mathrm{r}=.319$, $\mathrm{p}<.05$ ). This means that an increase in employee stress will lead to a decrease in health and safety and a decrease in employee stress will lead to an increase in health and safety.

The independent t-test was used to analyze this hypothesis that workers in the production section will experience lower health and safety compared to workers in the non-production section as mean scores of two independent groups were compared. Results are presented on Table 3 below.

Table 3. Section of work and worker health and safety

\begin{tabular}{lllllll}
\hline Section & N & Mean & SD & df & $\boldsymbol{t}$ & $\boldsymbol{p}$ \\
\hline Production & 66 & 21.65 & 2.03 & 118 & .45 & .38 \\
Non-Production & 54 & 20.14 & 3.13 & & & \\
\hline
\end{tabular}

From Table 3, the mean and standard deviation of health and safety level of employees in the production sector is $(\mathrm{M}=21.65, \mathrm{SD}=2.03)$ and that of employees in the non-production section is $(\mathrm{M}=20.14, \mathrm{SD}=3.13)$. The independent $t$-test results showed that, the effect of section of work on employee health and safety is not significant $\left(\mathrm{t}_{(118)}=.45, p=\mathrm{ns}\right)$.

The third hypothesis was tested using Independent $t$ test because two independent scores were compared. The results are summarized in Table 4 below.

Table 4. Type of shift work on worker health and safety

\begin{tabular}{lllllll}
\hline Shift & N & Mean & SD & $d f$ & $t$ & $p$ \\
\hline Shift work & 85 & 64.55 & 7.16 & 118 & 5.63 & .000 \\
No shift work & 35 & 24.70 & 12.13 & & & \\
\hline
\end{tabular}

From Table 4, there is a difference in the mean and standard deviation of health and safety of employees who run on shift $(M=64.55, S D=7.16)$ and that of employees who do not run on shift $(M=24.70, S D=12.13)$. The independent $t$ test results showed that the effect of type of shift on employee health and safety is significant $\left(\mathrm{t}_{(118)}=.01, \mathrm{p}<.05\right)$. Therefore the third hypothesis that states that employees who go through shift work will experience lower health and safety compared to employees who do not go through shift work was supported.

\subsection{Summary of Main Findings}

1) There was a significant negative relationship between employee stress and health and safety.

2) No significant difference was found in the level of health and safety among workers in the production sectors compared to workers in the non-production sectors.

3) Employees who go through shift work were found to experience lower health and safety compared to employees who do not go through shift work.

\section{Discussion}

The present study examined job stress, sector of work and shift work pattern as correlates of worker health and safety in a manufacturing company inGhana. Danquah (2002) emphasized that no human is stress-free in life and that stress is unavoidable. The first hypothesis that stated that there will be a significant negative relationship between employee stress and health and safety was supported. This means that as employee stress increases, the level of health declines and employees are likely be involved in accident. This finding is consistent with many previous findings (Barling, 1990; Chan, Lai, Ko, \& Boey, 2000; Piko, 1999; Plante \& Bouchard, 1995).Chan, Lai, Ko, and Boey (2000) especially assessed the relationship between stress, health and safety among 
employees of a manufacturing companies. They found that the level of stress correlates negatively with employee's health. The study again found a significant negative relationship between stress and the amount of accident that occurred.The significant negative relationship between stress of employees and their level of health and safety can also be explained with the Multidimensional Health Theory (Martens, 2001). According to this theory, psychological health is multidimensional in nature, composed of a cognitive component and a somatic component. Per the multidimensional theory, in the case of cognitive state anxiety, stress and depression, as anxiety increase, performance and health begins to deteriorate. Majority of the sample for the present study were engaged in the production section, where alertness, attention and good coordination are required. This may demand some amount of cognitive strength leading to the stress. This likely explains why a negative relationship was found between stress of the manufacturing employees and worker health and safety.

The second hypothesis that workers in the production sectors will experience lower health and safety compared to workers in the non-production sectors was not supported. This means that employee do not differ in the level of health and safety whether they work at the production or non-production stage. The present finding agrees with many researchers (Chan, Lai, Ko, \& Boey, 2000; Iqbal \& Kokash, 2011; Piko, 1999). These researchers found no significant difference in the level of stress and health among production and non-production sector employees. This finding is quite not surprising as employees in both production and non-production sectors are required by current organizational demands to go beyond their normal job description by performing many acts of citizenship behaviour (Agyemang, 2013; Badu \& Asumeng, 2013) leading to stress and lower health and safety. The inconsistency between the present study and that of Gmelch, Wilke and Lovrich (1996) can be due to the fact that Gmelch, Wilke and Lovrich (1996) used university employees whilst the present study used manufacturing employees. It is likely some other cultural factors and organizational factors may have influenced the findings of Gmelch, Wilke and Lovrich (1996). The inconsistency of the present finding with that of Gmelch, Wilke and Lovrich (1996) who indicated that production sector employees experience higher level of stress and lower health compared to employees in the non-production section should ignite more research to confirm or otherwise contingent on culture and sample. This is especially recommended as there is a high paucity of literature on this issue.

The third hypothesis that employees who go through shift work will experience lower health and safety compared to employees who do not go through shift work was supported. This means that the level of health is low for employees who run on shift compared to those who do not.

This finding implies that night shift working have a significant effect on employee wellbeing and arguably their personal and social life. Prolonged shift work, especially night shift work, also has a health risk as it produces symptoms that correspond closely to those of mild or moderate distress (Efinger, 1995). The present finding from the Ghanaian setting is consistent with some past findings (Akerstedt, 2007; Demi, 2006; Giovani, 1996; Zamanian, Kakooei, Ayattollahi, Karimian \& Seraji, 2008). Night shift work was significantly associated with anxiety more than morning and afternoon shift work. A number of reasons may have accounted for why shift workers experienced lower health and safety compared to employees who do not go. Shift workers and night workers often get tired and sleepy because of their work schedule. Being overly tired makes it difficult to concentrate, which increases the possibility of errors or accidents. This can be a risk both to the worker and other workers. Working at night makes it difficult to get enough sleep. Brain and body functions slow down during the night time and early morning hours. This makes employees less active and reduces performance and increases the risk of accidents (Smith, Folkard, Tucker \& Macdonald, 1994). In agreement with Akerstedt (1995), when working in the night shift, a person is at work when his or her circadian rhythm is low and asleep when it is high. Such a schedule means that a person is trying to stay alert when the circadian rhythm is low. This low-point affects physical activity and the ability to concentrate. If a worker also has lost sleep, fatigue could combine with the circadian low-point to double the effect on one's ability to perform. It is therefore not surprising that there was a ripple effect of shift workers and worker health and safety in the Ghanaian setting

\subsection{Limitations of the Study and Future Research Directions}

This present study like other studies is not devoid of limitations. It was very difficult getting a sample size that was equal in age, gender, and equal junior and senior staff for the study. The relatively small sample size limits the extent of generalizing the study findings. It is recommended that future researchers adopt a comparative approach that compares two organizations with one that run total shift and the other that do not run shift to ascertain whether there might be parity in findings contingent on the environment. It is likely other factors relating to the work situation may affect employee health and safety. It is recommended that these many unknown factors be unravelled through research. The researchers recommend that future researchers consider the 
extent to which an employee's psychological capital will moderate the relationship between stress and worker health and safety.

\subsection{Implications and Contributions of the Study}

The present study is an attempt to bridge the gap in the paucity of literature on how stress and sector of operation correlates with worker health and safety. The researchers sampled manufacturing workers and veers off from testing health and safety with frequently used medical workers. The study finding on section of operation widens the scholarly debate and deepens the woolliness and inconsistency of findings and spurs many future scholars into this area of research. The study implies that distress arising from the workplace varies from one working condition to the other. Support services should be preventative, so that health problems for employees can be averted. This requires more research into identifying the most effective way of detecting when individuals are experiencing early difficulties, and of improving their stress management techniques so as to prevent the transition to severe distress.

In conclusion, the present study findings offer an empirical backbone to a developing country like Ghana and many other developing countries with weak and fragmented health and safety policy bodies. Employers and employees alike must find ways to manage the likely antecedents of low worker health and safety. Adequate measures must be taken to control the amount of stress in work settings. It is recommended that employers take necessary steps towards introducing a culture where stress prevention plan exists, and stress is acknowledged and managed as part of everyday routine. This is a necessary step to curb the fragmentations of health and safety bodies where different industries have different health and safety policies. The researchers thus recommends that a health and safety authority be instituted in most developing countries to regulate health and safety issues at all levels.

\section{References}

Agyei, S. (2009). Work Stress and its Management among Senior Staff of the University of Cape Coast. Master of Philosophy Thesis. University of Cape Coast, Ghana.

Agyemang, C. B. (2013). Perceived Organizational Climate and Organizational Tenure on Organizational Citizenship Behaviour: Empirical Study among Ghanaian Banks'. European Journal of Business and Management, 5(26), 132-142.

Akerstedt, T. (1995). Work hours, sleepiness and accidents: introduction and summary. Journal of Sleep Research, 4(2), 1-3. http://dx.doi.org/10.1111/j.1365-2869.1995.tb00219.x

Alberta, S. (2008).Safety Culture Assessment: A Mission Impossible? Journal of Contingencies and Crisis Management, 17(4), 242-254.

Badu, A. C., \& Asumeng, M. (2013).Perceived Organizational Justice and Organizational Citizenship Behaviour in Ghana. European Journal of Business and Management, 5(19), 144-150.

Barling, R. S. (1990). The impact of working with people with HIV/AIDS: A review of the Literature. Social Science Medicine, 39, 221-232.

Baum, K., Singer, S., \& Baum, H. (1981). Stress and job satisfaction: A study of English Primary School. Educational Pyschology, 15(4), 473.

Beehr, T. A., \& Newman, J. E. (2006). Job Stress, Employee Health, and Organizational Effectiveness: A Facet Analysis, Model, and Literature Review. Personnel Psychology, 31(4), 665-699. http://dx.doi.org/10.1111/j.1744-6570.1978.tb02118.x

Bellani, M. L., Furlani, F., Gechi, M., \& Pezzolta, P. (1996).Burnout and related factors among HIV/AIDS health care workers. AIDS Care, 8(2), 207-215. http://dx.doi.org/10.1080/09540129650125885

Bennett, N., \& Kelaher, Y. (1994). Longitudinal predictors of burnout in HIV/AIDS health professional. Australian Journal of Public Health,18, 334-336. http://dx.doi.org/10.1111/j.1753-6405.1994.tb00255.x

Bless, P., \& Higson-Smith, T. (2000). Introduction to Health and Safety In Construction Industry (3rd ed.). B USA: utterworth-Heinemann, Imprint of Elsevier.

Buckingham, M., \& Saunders, P. (2004). Immune dysfunction associated with chronic professional stress in nurses. Psychiatry Research, 85, 105-111.

Chan, W., Lai, D., Ko, D., \& Boey, P. (2000). Stress, appraisal, and coping. New York: Springer.

Chaplain, R. P. (1995). Stress and job satisfaction: A study of English Primary School. Educational Psychology, 15(4), 473. http://dx.doi.org/10.1080/0144341950150409 
Cooper, C. L., \& Mitchell, S. J. (1990). Nursing the critically ill and dying. Human Relations, 43, 297-311. http://dx.doi.org/10.1177/001872679004300401

Costa, G. (1997). The problem shift works. Chronobiol International, 14, 89-98. http://dx.doi.org/10.3109/07420529709001147

De-Gucht, B. F., \& Demanet, C. (1990). Immune dysfunction associated with chronic professional stress in nurses. Psychiatry Research, 85, 105-111. http://dx.doi.org/10.1016/S0165-1781(98)00131-0

Demi, L. (2006). Exploration the burnout situation on teachers in two schools in maccau. NY: Journal article Report-Research.

Durand, H. B. (2003). Essentials of Abnormal Psychology (3rd ed.). Thompson Wadworth. USA: Pacific Grove.

Fimian, E., Fastenau, P., \& Thomas, H. (2009). The problem shift works. Chronobiol International, 14, 89-98.

Fimian, M. J., Fastenau, P. S., \& Thomas, J. (2009). Stress in nursing and intentions to leave the profession. Psychological Reports, 62, 499-506. http://dx.doi.org/10.2466/pr0.1988.62.2.499

Frank, M., \& Minh, P. (2007). A comparison of burnout in selected occupational fields. Career Department Quarterly, 38(3), 230-239.

Giovani, C. (1996). The impact of shift and night work on health. Applied Ergonomics, 27, 1.

Gmelch, D., Wilke, P., \& Lovrich, E. (1996). Psychosocial stress and cancer. Chichester, John Wily and Sons.

International Nuclear Safety Advisory Group (INSAG).(1986). Poor safety culture as one of the contributing factors to this worst nuclear power plant accident in history.

Iqbal, M., \& Kokash, M. (2011). Faculty Perception of Stress and Coping Strategies in a Saudi Private University: An Exploratory Study. International Education Studies, 4(3), 137-149. http://dx.doi.org/10.5539/ies.v4n3p137

Kinman, G., \& Jones, F. (2003).Running up the down escalator: stressors and strains in UK academics. Quality in Higher Education, 9(1), 22-37. http://dx.doi.org/10.1080/13538320308162

Kleiber, D. E., \& Guzy, B. (2003). Stress and burnout among health care personnel in the field of AIDS: Causes and prevalence. Amsterdam, University of Amsterdam Press.

Landsbergis, P. A. (2003). The Changing Organization of Work and the Safety and Health of working People: A Commentary. Journal of Occupational \& Environmental Medicine, 4(1), 61-72. http://dx.doi.org/10.1097/00043764-200301000-00014

Landsbergis, P. A. (2006). Assessing the contribution of working conditions to socioeconomic disparities in health: a commentary. Journal of Organizational Behavior, 9(3), 217-239. http://dx.doi.org/10.1002/job.4030090303

Lazarus, R., \& Folkman, S. (1984). Stress, appraisal, and coping. New York: Springer.

Letvak, S. (2005). Health and Safety among Older Nurses. Nursing Outlook, 53(2), 66-72. http://dx.doi.org/10.1016/j.outlook.2004.09.005

Martins, P. (2001). A comparison of burnout in selected occupational fields. Career Department Quaterly, 38(3), 230-239.

Oppenheim, A. N. (1992). Questionnaire Design, Interviewing and Attitude Measurement. London: Continuum.

Patton, M. Q. (1990). Qualitative evaluation and research methods (2nd ed.). Sage, Newbury Park, CA.

Piko, B. (1999). Work-Related stress among nurses: a challenge for health care institution. Journal of the Royal Society for the Promotion of Health, 119(3), 156-162. http://dx.doi.org/10.1177/146642409911900304

Plante, L. B. (1995). Occupational stress, burnout and professional support in nurses working with dying patients.

Reeves, S. L., Newling-Ward, E., \& Gissane, C. (2004). The effect of shift work on food intake and eating habits. Nutrition and Food Science, 34(5), 216-221. http://dx.doi.org/10.1108/00346650410560398

Reglin. G., \& Reitzammer, A. (1997). Dealing with the stress of teachers. Education, 4, 590-597.

Sanders, P. (2009). A Participative Approach to Developing Comprehensive Stress Management Interventions. In Occupational Health Psychology: Empowerment, Participation and Health at Work. European Academy of Occupational Health Psychology, 72-75. 
Spurgeon, A., Harrington, J. M.,\& Cooper, C. L. (1997).Health and safety problems associated with long working hours: a review of the current position. Occupational Environmental Medicine, 54,367-375.http://dx.doi.org/10.1136/oem.54.6.367

Tabachnick, B. G., \& Fidell, L. S. (1989). Using multivariate statistics (2nd ed.). New York: Harper \& Row.

Turk, D. C. (2002). A diathesis-stress model of chronic pain and disability following traumatic injury. Pain Research \& Management, 7(1), 9-20.

Tyler, F.,\& Ellison, B. (1994). Source of stress and Psychological Well Being on higher dependency nursing. Journal of Advance Nursing, 19, 469-476. http://dx.doi.org/10.1111/j.1365-2648.1994.tb01109.x

Wiersma, W., \& Jurs, S. (2005). Research Methods in Education (8th ed). Boston, MA: Pearson.

Willner, P. (1997). Validity, reliability and utility of the chronic mild stress model of depression: a 10 -year review and evaluation. Psychopharmacology, 134(4), 319-329. http://dx.doi.org/10.1007/s002130050456

Wu, W., Wang, M., \& Lan, Y. (2007). Relationship between burnout and occupational stress among nurses in China. Journal of Advanced nursing, 59(3), 233-239. http://dx.doi.org/10.1111/j.1365-2648.2007.04301.x

Yadama, G. N., \& Drake, B. (1995). Confirmatory factor analysis of the Maslach Burnout Inventory. Social Work Research, 19, 184-192.

Young, M.,\& Ogden, R. (2008). A survey of English speaking Canadian nurses in AIDS care. Journal of the Association of Nurses in AIDS Care, 9, 18-25. http://dx.doi.org/10.1016/S1055-3290(98)80057-5

Zamanian, R., Kakooei, D., Ayattollahi, W., Karimian S. M., \& Seraji, S. (2007). Mental Health Survey on Shift Work Nurses in Shiraz Province, Iran. Journal of School of Public Health and Institute of Public Health Research, 5, 47-54.

\section{Copyrights}

Copyright for this article is retained by the author(s), with first publication rights granted to the journal.

This is an open-access article distributed under the terms and conditions of the Creative Commons Attribution license (http://creativecommons.org/licenses/by/3.0/). 